\title{
Novel Controlled Criteria of Synchronization Stability in the Fractional Order Chen Attractor with Time Delays
}

\author{
Peizhong Liu*, Jianeng Tang, Chengti Huang and Lixin Zheng \\ College of Engineering, Huaqiao University, No.269, Chenghua North Road, \\ Fengze District, Quanzhou, Fujian362021, PR China \\ *Corresponding author's email:liupeizhong_1234@126.com
}

\begin{abstract}
In this paper, the chaotic behaviors in the fractional order Chen system with time delays are numerically investigated. By use of the fractional calculus techniques, we find that chaos exists in the fractional order Chen system with time delays when the order is less than 3. Chaossynchronization of the fractional order Chen system is theoretically and numerically studied using active control. Through the analysis of the error dynamical systems by using the Laplace transform theory, the time-delay correlative synchronization controller is designed to achieve chaos synchronization. At last, numerical simulations are provided to verify the effectiveness and feasibility of the developed method.
\end{abstract}

Keywords: fractional order; Chen system; chaos synchronization; the Laplace transform theory; time delays

\section{Introduction}

In the past 20 years, there has been a significant interest in developing powerful techniques for chaos synchronization since the pioneering work of Pecora and Carroll [1]. Several different regimes of chaos synchronization have been developed. They include partial synchronization [2], complete synchronization [3,4], phase synchronization [5, 6], generalized synchronization [7, 8], projective synchronization [9], and lag synchronization [10]. Many potential applications of chaos synchronization, especially in biological systems, chemical oscillators, secure communication, and electronic systems, have been discussed.

Delayed differential equations have been largely investigated in [11, 12], and references cited therein. Recently, time delays and time-varying delays are introduced to chaotic systems, e.g., see [13-16]. But most of these publications are for integer-order or typical differential equations. Although fractional calculus is a 300 -year-old mathematical topic, for many years it was not used in physics and engineering. However, during the last 10 years or so, fractional calculus starts to become a focus of interest of physicists and engineers from an application point of view [17, 18]. It has been found that many systems in interdisciplinary fields can be elegantly described by use of fractional derivatives. There are many known systems to display fractional-order dynamics, such as viscoelastic systems, dielectric polarization, electromagnetic waves, and quantum evolution of complex systems. Recently, it is a new trend that the fractional power of the control system dynamics [19-31].

In line with the Poincare-Bendixon theorem [19], we know that an integer order chaotic nonlinear system must have a minimum order of 3 for chaos to appear. However, it is not the case in the fractional order nonlinear systems. For example, it has been shown that the fractional order unified system of order as low as 2.76 can produce a chaotic attractor [20]. In [21], a striking finding is that the lowest order for the fractional-order Chen system to have chaos is 0.3. At the same time, J.G. Lu [22] found that the lowest order for chaos to be able to appear in the fractional-order Ikeda delay system was 0.1. By 
using Lambert function, the analytical stability bound is obtained for delayed secondorder systems with repeatable poles and the bound is obtained delayed linear timeinvariant fractional-order dynamic systems [23, 24]. Based on the Laplace transform theory, synchronization scheme to chaotic fractional-order Chen systems is derived in [25]; Deng et al. [26] introduced a characteristic equation for the n-dimensional linear fractional differential system with multiple time delays; the suitable conditions for achieving synchronization of the fractional order differential system are derived [20]. In line with the stability theorem of linear fractional systems, a necessary condition is given to check the chaos synchronization of fractional systems with incommensurate order [27]. In [28], Shao et al. proposed a method to achieve projective synchronization of the fractional order chaotic Rossler system. In [29], an analytical justification was proposed for phase synchronization of fractional differential equations. A stability test procedure was proposed for linear nonhomogeneous fractional order systems with a pure time delay [30]. Taghvafard and Erjaee [31] studied the phase and anti-phase synchronization between two identical and non-identical fractional order chaotic systems using techniques from active control theory. However, there are few results about chaos synchronization of the time-delay fractional order chaotic systems.

In our work, the chaotic behaviors in the fractional order Chen system with time delays are numerically investigated. By use of the fractional calculus techniques, we find that chaos exists in the fractional order Chen system with time delays when the order is less than 3. Chaossynchronization of the fractional order Chen system is theoretically and numerically studied using active control. Through the analysis of the error dynamical systems by using the Laplace transform theory, the time-delay correlative synchronization controller is designed to achieve chaos synchronization. At last, numerical simulations are provided to verify the effectiveness and feasibility of the developed method.

The organization of this paper is as follows. In Section 2, some definitions and systems are given and then an improved version of Adams-Bashforth-Moulton method is introduced. In Section 3, the time-delay correlative synchronization controller is designed to achieve chaos synchronization through the analysis of the error dynamical systems. The effectiveness of our work is verified through numerical examples in Section 4. Finally concluding remark is given.

\section{Definitions and Systems}

There are several definitions of a fractional-order differential system. In the following, we introduce the most common one of them:

$D_{*}^{\alpha} x(t)=J^{m-\alpha} x^{(m)}(t)$,

where $m=[\alpha]$, i.e., $m$ is the first integer which is not less than $\alpha(\alpha>0), x^{(m)}$ is the $m$ order derivative in the usual sense, and $J^{\beta}(\beta>0)$ is the $\beta$-order Reimann-Liouville integral operator with expression:

$J^{\beta} y(t)=\frac{1}{\Gamma(\beta)} \int_{0}^{t}(t-\tau)^{\beta-1} y(\tau) d_{\tau}$.

Here $\Gamma$ stands for Gamma function, and the operator $D_{*}^{\alpha}$ is generally called " $\alpha$-order Caputo differential operator" [32].

In 1999, Chen and Ueta [33] introduced a new chaotic system, which is similar but not topologically equivalent to the Lorenz system. The system is described by 


$$
\left\{\begin{array}{l}
\dot{x}=a(y-x), \\
\dot{y}=(c-a) x-x z+c y, \\
\dot{z}=x y-b z,
\end{array}\right.
$$

where $\mathrm{a}, \mathrm{b}$ and $\mathrm{c}$ are real parameters. When $a=35, b=3$ and $c=28$, system (3) displays a chaotic attractor, as shown in Figure 1. Without changing the model structure of the system, we consider the time-delay Chen system in the following form:

$$
\left\{\begin{array}{l}
\dot{x}=a(y-x) \\
\dot{y}=(c-a) x-x z+c y \\
\dot{z}=x y-b z(t-\tau(t))
\end{array}\right.
$$

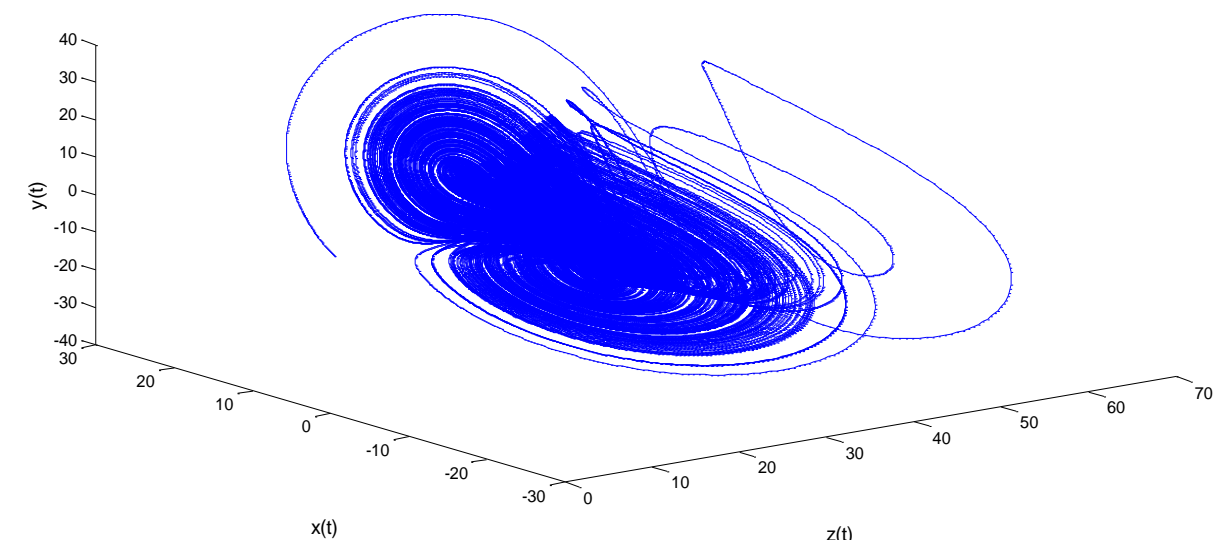

Figure 1. (Color Online);the Chen Chaotic attractors with $a=35, b=3, c=28$

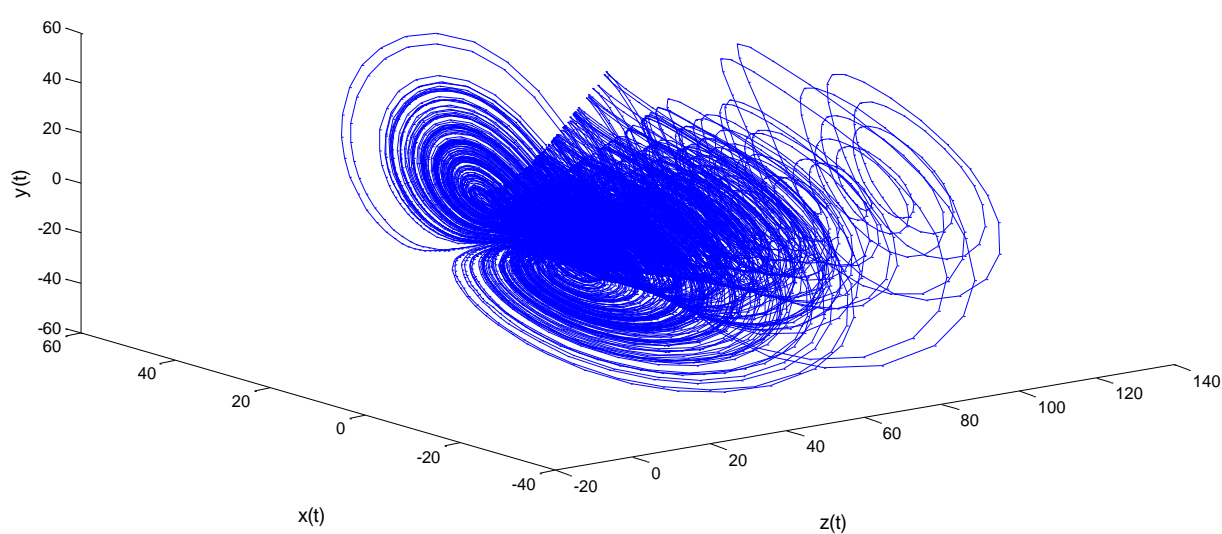

Figure 2. (Color Online); the Chen Chaotic Attractors with $a=35, b=3, c=28$ and $\tau=0.2$

where $\tau(t)\left(0 \leq \tau(t) \leq \tau_{m}\right)$ may be an unknown time-varying delay or a constant delay. The system (4) may be in the chaotic state through the suitable selection of time delay $\tau(t)$. When $\tau(t)=0.2$, system (4) displays a chaotic attractor as shown in Figure 2. 
Now, let us introduce its fractional versions as follows:

$$
\left\{\begin{array}{l}
\frac{d^{\alpha} x}{d t^{\alpha}}=a(y-x), \\
\frac{d^{\alpha} y}{d t^{\alpha}}=(c-a) x-x z+c y, \\
\frac{d^{\alpha} z}{d t^{\alpha}}=x y-b z
\end{array}\right.
$$

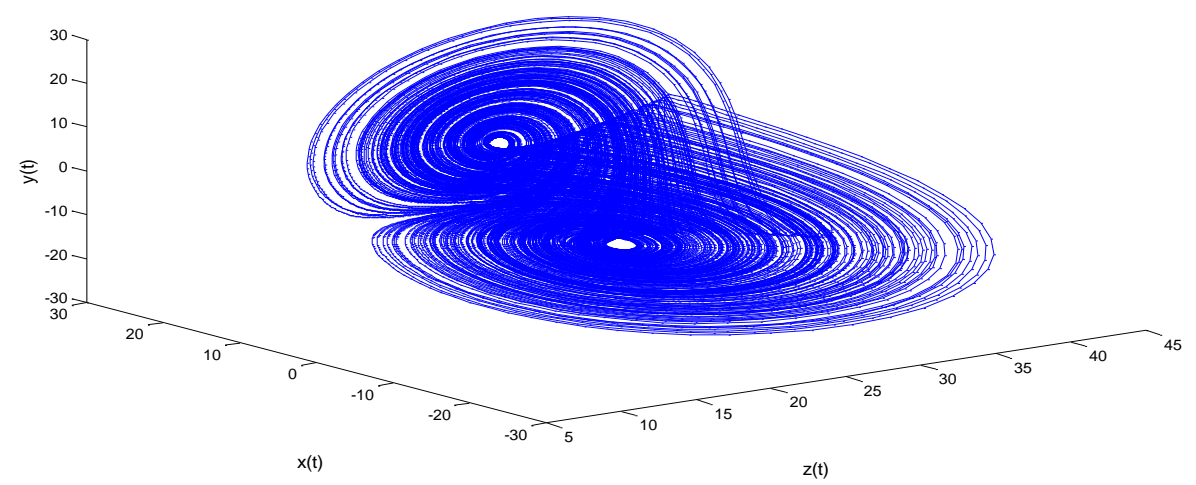

Figure 3.(Color Online); the Fractional-order Chen Chaotic Attractors with $a=35, b=3, c=28$ and $\alpha=0.98$

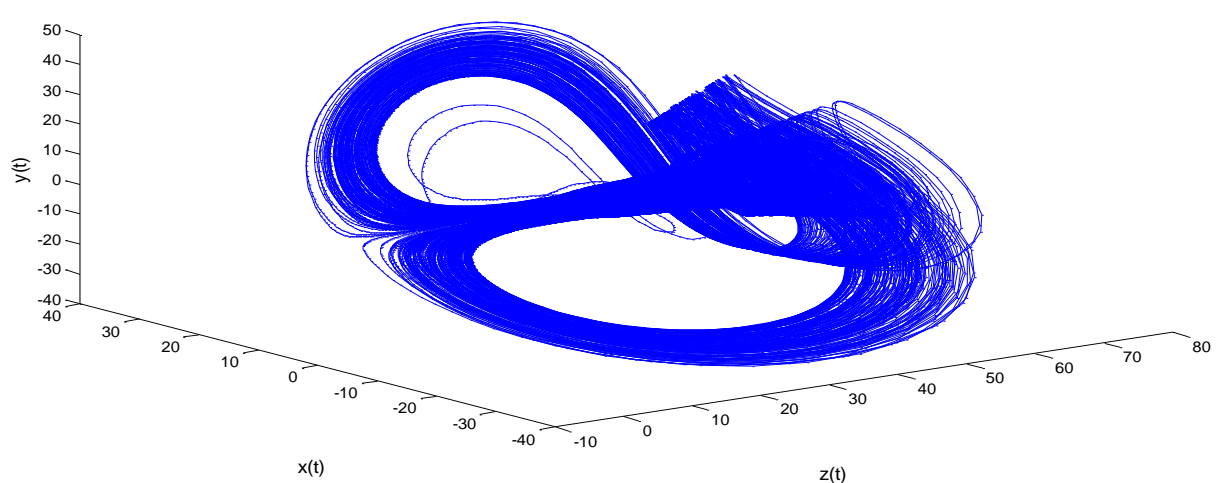

Figure 4.(Color Online); the Fractional-order Chen Chaotic Attractors with $a=35, b=3, c=28, \alpha=0.98$ and $\tau=0.2$

and

$\left\{\begin{array}{l}\frac{d^{\alpha} x}{d t^{\alpha}}=a(y-x), \\ \frac{d^{\alpha} y}{d t^{\alpha}}=(c-a) x-x z+c y, \\ \frac{d^{\alpha} z}{d t^{\alpha}}=x y-b z(t-\tau),\end{array}\right.$ 
where $0<\alpha \leq 1$. The lowest order to have chaos in system (5)was 2.76 in [20]. Here we have found that for a set of parameter values: $\mathrm{a}=35, \mathrm{~b}=3, \mathrm{c}=28$ and $\alpha=0.95$, the fractional-order Chen system can display chaotic attractors as shown in Figure3. When $\tau(t)=0.2$, the fractional-order Chen system with a constant delay also has a chaotic attractor as shown in Figure 4.

According to [34-36], the predictor-corrector scheme for system (6) is derived. This scheme is the generalization of Adams-Bashforth-Moulton one [36].

The following differential equation:

$$
\begin{array}{ll}
\frac{d^{\alpha} x}{d t^{\alpha}}=f(t, x), & 0 \leq \mathrm{t} \leq \mathrm{T}, \\
x^{(\mathrm{k})}(0)=x_{0}^{(k)}, & \mathrm{k}=0,1,2, \ldots, \mathrm{n}-1,
\end{array}
$$

is equivalent to the Volterra integral equation [35].

$$
x(t)=\sum_{k=0}^{n-1} x_{0}^{(k)} \frac{t^{k}}{k !}+\frac{1}{\Gamma(\alpha)} \int_{0}^{t} \frac{f(\tau 1, x)}{(t-\tau)^{1-\alpha}} d \tau 1 .
$$

Let $h=T / N, t_{n}=n h(n=0,1,2, \ldots, N), n 1=\tau / h$. Then Eq. (7) can be discretized as follows:

$x_{h}\left(t_{n+1}\right)=\sum_{k=0}^{n-1} x_{0}^{(k)} \frac{t_{n}^{k+1}}{k !}+\frac{h^{\alpha}}{\Gamma(\alpha+2)} f\left(t_{n+1}, x_{h}^{\rho}\left(t_{n+1}\right)\right)+\frac{h^{\alpha}}{\Gamma(\alpha+2)} \sum a_{j, n+1} f\left(t_{j}, x_{h}\left(t_{j}\right)\right)$,

where

$a_{j, n+1}= \begin{cases}n^{\alpha+1}-(n-\alpha)(n+1)^{\alpha+1}, & \mathrm{j}=0 \\ (n-j+2)^{\alpha+1}+(n-j)^{\alpha+1}-2(n-j+1)^{\alpha+1}, & 1 \leq \mathrm{j} \leq \mathrm{n} \\ 1, & \mathrm{j}=\mathrm{n}+1\end{cases}$

$x_{h}^{\rho}\left(t_{n+1}\right)=\sum_{k=0}^{n-1} x_{0}^{(k)} \frac{t_{n}^{k+1}}{k !}+\frac{1}{\Gamma(\alpha)} \sum_{j=0}^{n} b_{j, n+1} f\left(t_{j}, x_{h}\left(t_{j}\right)\right), b_{j, n+1}=\frac{h^{\alpha}}{\alpha}\left((n-j+1)^{\alpha}-(n-j)^{\alpha}\right)$.

The error estimate is $e=\operatorname{Max}\left|x\left(t_{j}\right)-x_{h}\left(t_{j}\right)\right|=O\left(h^{\rho}\right)(j=0,1, \ldots, N) \quad$ where $\rho=\operatorname{Min}(2,1+\alpha)$.

Applying the above method, Eq. (6) can be discretized as follows:

$$
\left\{\begin{array}{l}
x_{n+1}=x_{0}+\frac{h^{\alpha}}{\Gamma(\alpha+2)} a\left(y_{n+1}^{\rho}-x_{n+1}^{\rho}\right)+\frac{h^{\alpha}}{\Gamma(\alpha+2)} \sum_{\mathrm{j}=0}^{\mathrm{n}} \gamma_{1, \mathrm{j}, \mathrm{n}+1} a\left(y_{j}-x_{j}\right), \\
y_{n+1}=y_{0}+\frac{h^{\alpha}}{\Gamma(\alpha+2)}\left[(c-a) x_{n+1}^{\rho}-x_{n+1}^{\rho} z_{n+1}^{\rho}+c y_{n+1}^{\rho}\right]+\frac{h^{\alpha}}{\Gamma(\alpha+2)} \sum_{\mathrm{j}=0}^{\mathrm{n}} \gamma_{2, \mathrm{j}, \mathrm{n}+1}\left[(c-a) x_{j}-x_{j} z_{j}+c y_{j}\right], \\
z_{n+1}=z_{0}+\frac{h^{\alpha}}{\Gamma(\alpha+2)}\left(x_{n+1}^{\rho} y_{n+1}^{\rho}-b z_{n+1-n)}^{\rho}\right)+\frac{h^{\alpha}}{\Gamma(\alpha+2)} \sum_{\mathrm{j}=0}^{\mathrm{n}} \gamma_{3, \mathrm{j}, \mathrm{n}+1}\left(x_{j} y_{j}-b z_{j-n 1}\right),
\end{array}\right.
$$


where

$$
\begin{aligned}
& x_{n+1}^{\rho}=x_{0}+\frac{1}{\Gamma(\alpha+2)} \sum_{\mathrm{j}=0}^{\mathrm{n}} \omega_{1, \mathrm{j}, \mathrm{n}+1} a\left(y_{j}-x_{j}\right), \\
& y_{n+1}^{\rho}=y_{0}+\frac{1}{\Gamma(\alpha+2)} \sum_{\mathrm{j}=0}^{\mathrm{n}} \omega_{2, \mathrm{j}, \mathrm{n}+1}\left[(c-a) x_{j}-x_{j} z_{j}+c y_{j}\right], \\
& z_{n+1}^{\rho}=z_{0}+\frac{1}{\Gamma(\alpha+2)} \sum_{\mathrm{j}=0}^{\mathrm{n}} \omega_{3, \mathrm{j}, \mathrm{n}+1}\left(x_{j} y_{j}-b z_{j-n 1}\right), \\
& \gamma_{i, j, n+1}= \begin{cases}n^{\alpha+1}-(n-\alpha)(n+1)^{\alpha+1}, & \mathrm{j}=0 \\
(n-j+2)^{\alpha+1}+(n-j)^{\alpha+1}-2(n-j+1)^{\alpha+1}, & 1 \leq \mathrm{j} \leq \mathrm{n} \\
1, & \mathrm{j}=\mathrm{n}+1\end{cases} \\
& \omega_{i, j, n+1}=\frac{h^{\alpha}}{\alpha}\left((n-j+1)^{\alpha}-(n-j)^{\alpha}\right), \quad 0 \leq j \leq n, i=1,2,3 .
\end{aligned}
$$

\section{Design of Chaotic Synchronization Controller}

The main title (on the first page) should begin $13 / 16$ inches ( 7 picas) from the top edge of the page, centered, and in Times New Roman 14-point, boldface type. Capitalize the first letter of nouns, pronouns, verbs, adjectives, and adverbs; do not capitalize articles, coordinate conjunctions, or prepositions (unless the title begins with such a word). Please initially capitalize only the first word in other titles, including section titles and first, second, and third-order headings (for example, "Titles and headings" — as in these guidelines). Leave two blank lines after the title.

In the section, based on the nonlinear control theory, an effective method for designing synchronization methods in the fractional order Chen chaotic systems with time delays has proposed to realize synchronization.

The chaos synchronization consists in the tracking of the master system trajectories by the slave system. However, this problem can be restated as the stabilization of the orbits of a dynamical system which represents the discrepancy between two systems. In order to observe the synchronization behavior in two identical fractional-order Chen systems with time delays, we build a drive-response configuration with a drive system given by the fractional-order Chen system with time delays (with three state variables denoted by the subscript $\mathrm{m}$ ) and with a response system (with three variables denoted by subscript $\mathrm{s}$ ). The drive and response systems are described by the following differential equations, respectively:

$$
\left\{\begin{array}{l}
\frac{d^{\alpha} x_{m}}{d t^{\alpha}}=a\left(y_{m}-x_{m}\right), \\
\frac{d^{\alpha} y_{m}}{d t^{\alpha}}=(c-a) x_{m}-x_{m} z_{m}+c y_{m}, \\
\frac{d^{\alpha} z_{m}}{d t^{\alpha}}=x_{m} y_{m}-b z_{m}(t-\tau),
\end{array}\right.
$$


and

$\left\{\begin{array}{l}\frac{d^{\alpha} x_{s}}{d t^{\alpha}}=a\left(y_{s}-x_{s}\right)+u_{1}(t) \\ \frac{d^{\alpha} y_{s}}{d t^{\alpha}}=(c-a) x_{s}-x_{s} z_{s}+c y_{s}+u_{2}(t) \\ \frac{d^{\alpha} z_{s}}{d t^{\alpha}}=x_{s} y_{s}-b z_{s}(t-\tau)+u_{3}(t)\end{array}\right.$

where $U=\left[u_{1}(t), u_{2}(t), u_{3}(t)\right]^{T}$ is the nonlinear controller to be designed. Our aim is to determine the controller $\mathrm{U}$ for the global synchronization of two identical fractional-order Chen systems with time delays. For this purpose, we define the synchronization error as follows:

$e_{1}=x_{s}-x_{m}, e_{2}=y_{s}-y_{m}, e_{3}=z_{s}-z_{m}$,

Then subtracting system (9) from system (10) yields the following error system:

$\left\{\begin{array}{l}\frac{d^{\alpha} e_{1}}{d t^{\alpha}}=a\left(e_{2}-e_{1}\right)+u_{1}(t), \\ \frac{d^{\alpha} e_{2}}{d t^{\alpha}}=(c-a) e_{1}-e_{1} e_{3}-x_{m} e_{3}-z_{m} e_{1}+c e_{2}+u_{2}(t), \\ \frac{d^{\alpha} e_{3}}{d t^{\alpha}}=e_{1} e_{2}+x_{m} e_{2}+y_{m} e_{1}-b e_{3}(t-\tau)+u_{3}(t) .\end{array}\right.$

Now, we define the control functions as follows:

$\left\{\begin{aligned} u_{1}(t) & =-a\left(e_{2}-e_{1}\right)+a_{11} e_{1}+b_{11} e_{1}(t-\tau)+a_{12} e_{2}+b_{12} e_{2}(t-\tau), \\ u_{2}(t) & =-(c-a) e_{1}+e_{1} e_{3}+x_{m} e_{3}+z_{m} e_{1}-c e_{2}+a_{22} e_{2}+b_{22} e_{2}(t-\tau), \\ u_{3}(t) & =-e_{1} e_{2}+\left(a_{31}-y_{m}\right) e_{1}+b_{31} e_{1}(t-\tau) \\ & +\left(a_{32}-x_{m}\right) e_{2}+b_{32} e_{2}(t-\tau)+a_{33} e_{3}+\left(b_{33}-b\right) e_{3}(t-\tau) .\end{aligned}\right.$

Under this particular choice, the error system (11) becomes

$\left\{\begin{array}{l}\frac{d^{\alpha} e_{1}}{d t^{\alpha}}=a_{11} e_{1}+b_{11} e_{1}(t-\tau)+a_{12} e_{2}+b_{12} e_{2}(t-\tau), \\ \frac{d^{\alpha} e_{2}}{d t^{\alpha}}=a_{22} e_{2}+b_{22} e_{2}(t-\tau), \\ \frac{d^{\alpha} e_{3}}{d t^{\alpha}}=a_{31} e_{1}+b_{31} e_{1}(t-\tau)+a_{32} e_{2}+b_{32} e_{2}(t-\tau)+a_{33} e_{3}+b_{33} e_{3}(t-\tau) .\end{array}\right.$

Let

$$
\bar{D}_{*}^{\alpha} e=\left[\frac{d^{\alpha} e_{1}}{d t^{\alpha}}, \frac{d^{\alpha} e_{2}}{d t^{\alpha}}, \frac{d^{\alpha} e_{3}}{d t^{\alpha}}\right]^{\mathrm{T}},
$$

Then the error system (13) can be written as

$$
\bar{D}_{*}^{\alpha} e=A e+B e(t-\tau)
$$

where $e=\left[e_{1}, e_{2}, e_{3}\right]^{\mathrm{T}}, e(t-\tau)=\left[e_{1}(t-\tau), e_{2}(t-\tau), e_{3}(t-\tau)\right]^{\mathrm{T}}$ and 


$$
A=\left[\begin{array}{lll}
a_{11} & a_{12} & 0 \\
0 & a_{22} & 0 \\
a_{31} & a_{32} & a_{33}
\end{array}\right], B=\left[\begin{array}{lll}
b_{11} & b_{12} & 0 \\
0 & b_{22} & 0 \\
b_{31} & b_{32} & b_{33}
\end{array}\right] .
$$

Theorem 1. For the error dynamical system (14), if the time-delay correlative control parameter of controller (12) and the time delay in system (9) satisfy

(1) $a_{i j}<b_{i j}<0,1 \leq i, j \leq 3$. (2) $\tau$ is a constant greater than zero.

That is, system (9) and system (10) are synchronized.

Proof. Taking the Laplace transformation in both sides of Eq.(13), letting $E_{i}(s)=L\left(e_{i}(t)\right)(i=1,2,3)$, and utilizing $L\left(d^{\alpha} e_{i} / d t^{\alpha}\right)=s^{\alpha} E_{i}(s)-s^{\alpha-1} e_{i}(0)$ (see Ref. $[18$, p. 70$])$, we obtain

$$
\left\{\begin{aligned}
s^{\alpha} E_{1}(s)-s^{\alpha-1} e_{1}(0) & =a_{11} E_{1}(S)+b_{11} E_{1}(s) e^{-s \tau}+a_{12} E_{2}(s)+b_{12} E_{2}(s) e^{-s \tau} \\
s^{\alpha} E_{2}(s)-s^{\alpha-1} e_{2}(0) & =a_{22} E_{2}(s)+b_{22} E_{2}(s) e^{-s \tau} \\
s^{\alpha} E_{3}(s)-s^{\alpha-1} e_{3}(0) & =a_{31} E_{1}(s)+b_{31} E_{1}(s) e^{-s \tau}+ \\
& a_{32} E_{2}(s)+b_{32} E_{2}(s) e^{-s \tau}+a_{33} E_{3}(s)+b_{33} E_{3}(s) e^{-s \tau}
\end{aligned}\right.
$$

Eq. (15) can be rewritten as follows:

$$
\left\{\begin{array}{l}
E_{1}(s)=\frac{s^{\alpha-1} e_{1}(0)+\left(a_{12}+b_{12} e^{-s \tau}\right) E_{2}(s)}{s^{\alpha}-a_{11}-b_{11} e^{-s \tau}} \\
E_{2}(s)=\frac{s^{\alpha-1} e_{2}(0)}{s^{\alpha}-a_{22}-b_{22} e^{-s \tau}} \\
E_{3}(s)=\frac{s^{\alpha-1} e_{3}(0)+\left(a_{31}+b_{31} e^{-s \tau}\right) E_{1}(s)+\left(a_{32}+b_{32} e^{-s \tau}\right) E_{2}(s)}{s^{\alpha}-a_{33}-b_{33} e^{-s \tau}}
\end{array}\right.
$$

By use of the final-value theorem of the Laplace transformation, we have

$\lim _{t \rightarrow+\infty} e_{2}(t)=\lim _{s \rightarrow 0^{+}} s E_{2}(s)=\frac{s^{\alpha} e_{2}(0)}{s^{\alpha}-a_{22}-b_{22} e^{-s \tau}}$,

If two conditions of the proposed theorem are satisfied, that is, $\tau>0$ and $a_{22}<b_{22}<0$, then we get

$\lim _{t \rightarrow+\infty} e_{2}(t)=\frac{s^{\alpha} e_{2}(0)}{s^{\alpha}-a_{22}-b_{22} e^{-s \tau}}=0$.

At the same time, from Eq.(16), we can get

$$
\begin{aligned}
& \lim _{t \rightarrow+\infty} e_{1}(t)=\lim _{s \rightarrow 0^{+}} s E_{1}(s)=\frac{s^{\alpha} e_{1}(0)+s\left(a_{12}+b_{12} e^{-s \tau}\right) E_{2}(s)}{s^{\alpha}-a_{11}-b_{11} e^{-s \tau}}=0 \\
& \lim _{t \rightarrow+\infty} e_{3}(t)=\lim _{s \rightarrow 0^{+}} s E_{3}(s)=\frac{s^{\alpha} e_{3}(0)+s\left(a_{31}+b_{31} e^{-s \tau}\right) E_{1}(s)+s\left(a_{32}+b_{32} e^{-s \tau}\right) E_{2}(s)}{s^{\alpha}-a_{33}-b_{33} e^{-s \tau}}=0
\end{aligned}
$$

The above analysis implies that the fractional-order drive system (9) and the fractionalorder response system (10) with the active control law (12) are synchronized. The proof is completed. 


\section{Numerical Results}

Based on the predictor-corrector scheme, we propose a numerical algorithm which can be applied to find numerical solutions of the systems (9) and (10). The above discretization scheme in Section 2 is an improved version of AdamsBashforth-Moulton method [36]. In the numerical simulations, the initial states of the drive system (9) and the response system (10) are taken as $x_{m}(0)=1, y_{m}(0)=3, z_{m}(0)=2$ and $x_{s}(0)=2, y_{s}(0)=4, z_{s}(0)=3$, respectively. Here we take $x_{m}(t)=1, y_{m}(t)=2, z_{m}(t)=3$ and $x_{s}(t)=3, y_{s}(t)=8, z_{s}(t)=15$ when $-\tau \leq t<0$. And the time delay $\tau$ is always chosen as $\tau=0.2$.

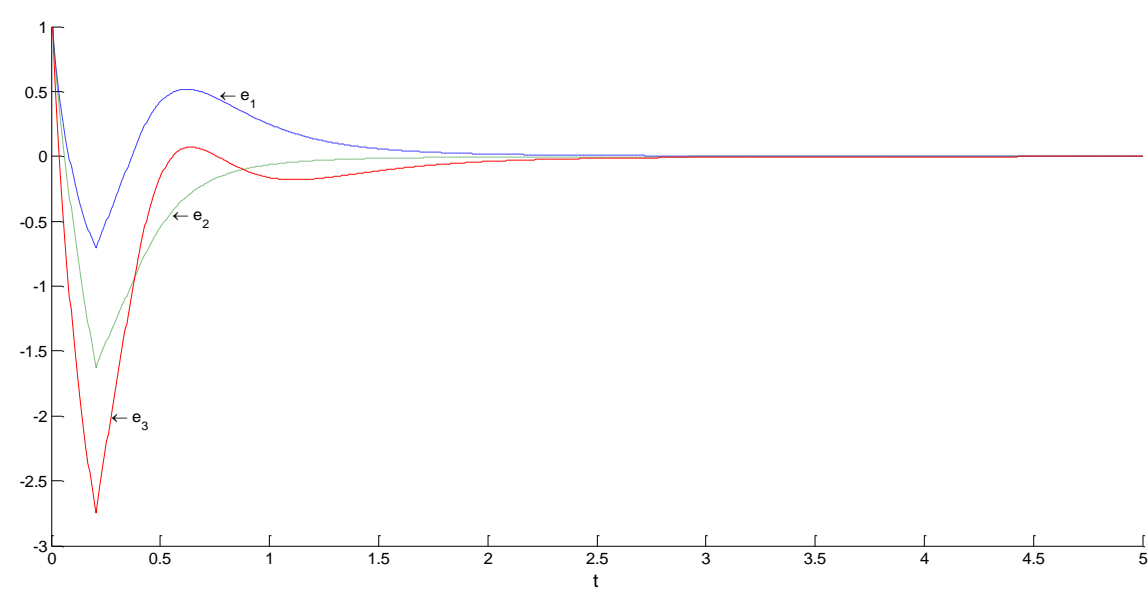

Figure 5. (Color Online);the Error Dynamics of Synchronization between System (9) and (10) with $\alpha=0.98$ and $\tau=0.2$

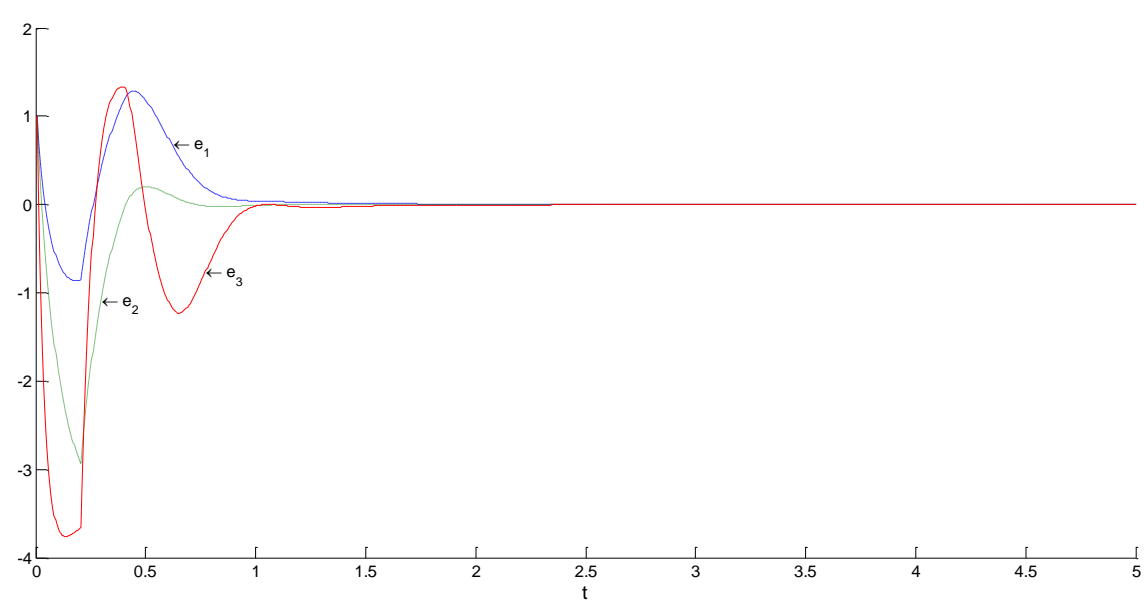

Figure 6. (Color Online); the Error Dynamics of Synchronization between System (9) and (10) with $\alpha=0.95$ and $\tau=0.2$

When

$$
A=\left[\begin{array}{llc}
-2 & -2 & 0 \\
0 & -2 & 0 \\
-2 & -2 & -2
\end{array}\right], B=\left[\begin{array}{ccc}
-1 & -1 & 0 \\
0 & -1 & 0 \\
-1 & -1 & -1
\end{array}\right]
$$


and the fractional order $\alpha=0.98$, the error system (14) is globally asymptotically stable according to the proposed theorem. Thus we can obtain the synchronization between system (9) and system (10). The error dynamics of synchronization of system (9) and system (10) are shown in Figure5. Seen from Figure5, the synchronization of system (9) and system (10) is achieved. Similarly, we obtain the synchronization between system (9) and system (10) when

$A=\left[\begin{array}{ccc}-3 & -5 & 0 \\ 0 & -8 & 0 \\ -10 & -6.5 & -15\end{array}\right], B=\left[\begin{array}{ccc}-1 & -2.5 & 0 \\ 0 & -2 & 0 \\ -8 & -5 & -3\end{array}\right]$

and $\alpha=0.95$. From Figure 6, the synchronization of system (9) and system (10) is also achieved.

\section{Conclusions}

Simulation of fractional order Chen attractors with time delays are discussed in this paper. Seen from the view point of these simulations, the chaotic attractors of fractional order Chen systems with time delays are different from the original Chen chaotic attractors. Based on the analysis of the error dynamical systems by using the Laplace transform theory, time-delay correlative controller is successfully applied to achieving synchronization. Numerical simulations are also given to show the effectiveness of the proposed method.

\section{Acknowledgments}

This work was supported by the Natural Science Foundation of China under Grant No. 61231002 and No. 51075068, by Cloud Computing Platform for Internet of Things-Fujian Scientific Research Platform for Innovation under Grant No. 2013H2002, by the Foundation of Huaqiao University under Grant No. 12BS228 and No. 13BS103. The authors would like to thank the reviewers for their valuable suggestions and comments.

\section{References}

[1] L. M. Pecora and T.L. Carroll, "Phys. Rev. Lett.", vol. 64, no. 821, (1990).

[2] R. Aguilar-López and R. Martinez-Guerra, "Chaos Solitons and Fractals", vol. 33, no. 572, (2007).

[3] J. N. Tang, C. R. Zou, S. P. Wang, L. Zhao and X. P. Liu, "Int. J. Bifurc. Chaos", vol.22, 1250183(2012)

[4] X. Wang and G. Chen, "Int. J. Bifurc. Chaos", vol. 12, no. 187, (2002).

[5] M.G. Rosenblum, A.S. Pikovsky and J. Kurths, "Phys. Rev. Lett.”, vol. 76, no. 1804, (1996).

[6] C. Sarasola, F.J. Torrealdea, A. d'Anjou and M. Graňa, "Math. Comput. Simulat.", vol. 58, no. 309, (2002).

[7] G. Zhang, Z. Liu, Z. Ma and Chaos, "Solitons and Fractals", vol. 32, no. 773, (2007).

[8] R. Brown, "Phys. Rev. Lett.", vol. 81, no.4835, (1998).

[9] R. Mainieri and J. Rehacek, "Phys. Rev. Lett.", vol. 82, no. 3042, (1999).

[10] X. Zhang, X. Liao and C. Li, "Chaos, Solitons and Fractals", vol. 26, no. 845, (2005).

[11] J. N. Tang, "Math. Probl. Eng.", 2014, 262151(2014).

[12] W.H. Deng, Y.J. Wu and C.P. Li, "Int. J. Bifurc. Chaos", vol.16, no. 465, (2006).

[13] C.-K. Cheng, H.-H. Kuo, Y.-Y. Hou, C.-C. Hwang and T.-L. Liao, "Physica A", vol. 387, no. 3093, (2008).

[14] Y.Z. Xiao, W. Xu, S.F. Tang and X.C. Li, “Applied Mathematics and Computation”, vol. 213, no. 547, (2009).

[15] W.L. He, F. Qian, J.D. Cao and Q.-L. Han, "Phys. Lett. A", vol. 375, no. 498, (2011).

[16] J.P. Richard, "Automatica", vol. 39, no. 1667, (2003).

[17] I. Podlubny, "Fractional Differential Equations", Academic Press, New York (1999).

[18] R. Hifer, “Applications of Fractional Calculus in Physics”, World Scientific, New Jersey (2001).

[19] M.W. Hirsch and S. Smale, "Differential Equations: Dynamical Systems and Linear Algebra", Academic Press, New York (1974).

[20] X.J. Wu, J. Li and G.R. Chen, “J. Franklin Institute”, vol. 345, no. 392, (2008). 
[21] J. G. Lu and G.R. Chen, "Chaos, Solitons and Fractals", vol. 27, no. 68, (2006).

[22] J. G. Lu, "Chin. Phys.", vol. 15, no. 301, (2006).

[23] Y.Q. Chen and K. L. Moore, “Automatica”, vol. 38, no. 891, (2002).

[24] Y.Q. Chen and K. L. Moore, "Nonlinear Dyn.”, vol. 29, no. 191, (2002).

[25] J.W. Wang, X.H. Xiong and Y.B. Zhang, "Physica A", vol. 370, no. 279, (2006).

[26] W.H. Deng, C.P. Li and J.H. Lü, "Nonlinear Dyn.", vol. 48, no. 409, (2007).

[27] H. Zhu, S.B. Zhou and Z.S. He, "Chaos, Solitons and Fractals", vol. 41, no. 2733, (2009).

[28] S.Q. Shao, X.Gao and X.W. Liu, "Chin.Phys.", vol. 16, no. 2612, (2007).

[29] G.H. Erjaee and S. Momani, "Phys. Lett. A", vol. 372, no. 2350, (2008).

[30] M. P. Lazarević, A. M. Spasić, "Mathematical and Computer Modelling”, vol. 49, no. 475, (2009).

[31] H. Taghvafard and G.H. Erjaee, "Commun. Nonlinear SciNumerSimulat.”, vol. 16, no. 4079, (2011).

[32] M. Caputo, “Geophys. J. R. Astron. Soc.”, vol. 13, no. 529, (1967).

[33] G.R. Chen and T. Ueta, "Int. J. Bifurc. Chaos", vol. 9, no. 1465, (1999).

[34] K. Diethelm, "Electron.Trans. Numer. Anal.”, vol. 5, no. 1, (1997).

[35] K. Diethelm and N.J. Ford, "J. Math. Anal. Appl.", vol. 265, no. 229, (2002).

[36] K. Diethelm, N.J. Ford and A.D. Freed, "Nonlinear Dyn.”, vol. 29, no. 3 (2002).

\section{Authors}

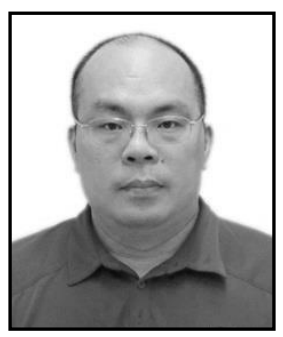

Peizhong Liu, he was born in 1976 . He received the P. H. degree from school of information science and engineering, Xiamen University, Xiamen, Fujian, China. Now he is an IOT Experimentalist, and his research interests include multi-dimensional space biomimetic informatics, visual media retrieval, network model, information security.

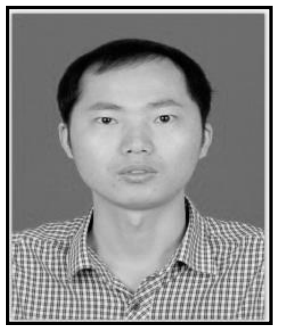

Jianeng Tang, he was born in 1983. He received the Ph. D. degree in Information and Communication Engineering from Southeast University in 2012. Currently, he is with College of Engineering, Huaqiao University, Quanzhou, Fujian, China. His main research interests include nonlinear systems, chaos synchronization and control, complex networks, acoustic emission technology and speech signal processing.

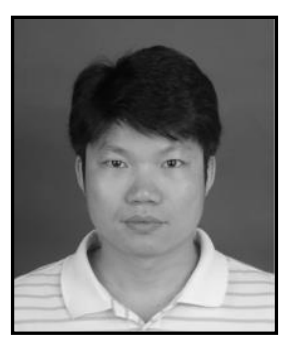

Chengti Huang, he was born in 1980. He received the P.H. degree from Automation Engineering, University of Electronic and Technology of China. His research interests include signal processing, electronic test and fault diagnosis, communication.

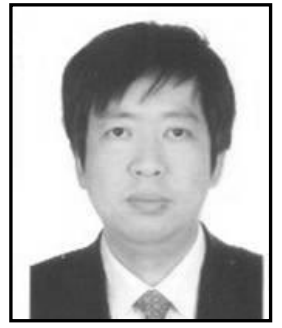

Lixin Zheng, he was born in 1967.He received the P.H. degree from the College of Precision Measuring Technology \& Instruments, Tianjin University, Tianjin 300072, China. Now he is the head of the College of Industry, Huaqiao University, China and his research interests include 3D measurements, computer control and computer vision. 
International Journal of Control and Automation

Vol.8, No.5 (2015) 